Egyptian Journal of Aquatic Biology \& Fisheries

Zoology Department, Faculty of Science,

Ain Shams University, Cairo, Egypt.

ISSN $1110-6131$

Vol. 24(1): $337-347$ (2020)

www.ejabf.journals.ekb.eg

\title{
Evaluation of morphometric and molecular variations among some Egyptian brine shrimps comparatively with other Artemia species
}

\section{Yasser M. Saad ${ }^{1,3^{*}}$ and Heba E. A. Elsebaie ${ }^{2}$}

1- Genetics Lab., National Institute of Oceanography and Fisheries, Cairo, Egypt.

2- Hydrobiology Lab., National Institute of Oceanography and Fisheries, Cairo, Egypt

3- Department of Biological Sciences, Faculty of Sciences, King Abdulaziz

University, Kingdom of Saudi Arabia.

*Corresponding author: yasser_saad19@yahoo.com

\section{ARTICLE INFO}

Article History:

Received: Dec. 10, 2019

Accepted: Dec. 28, 2019

Online: Jan. 2020

Keywords:

Artemia

Morphology

Genetics

Molecular

Variations

\section{ABSTRACT}

Up to date, the true phylogenetic relationships among the Artemia species are under debate. In this study, some morphometric and molecular (Inter-simple sequence repeats, ISSR) variations were analyzed to evaluate the biodiversity among some Egyptian Artemia species comparatively with other brine shrimps (A. parthenogenetica, A. salina and A. franciscana). The highest and lowest Wilks' lambda values were calculated for the Length of furca and the abdominal length. The cluster analysis based on the Artemia morphological variations was an agreement with the re-constructed dendrogram based on ISSR markers. The ISSR variations were comparatively analyzed with the Artemia species Cytochrome oxidase subunit I gene (COI) sequence variations. Based on the COI consensus sequences, the distance value between $A$. salina and $A$. parthenogenetica was higher than the distance value between A. salina and A. franciscana. The ISSR could be an effective method in Artemia molecular characterization and evolutionary studies. The results could be helpful in the conservation of the evaluated Artemia species. The combination of more informative molecular markers with the selected morphometric characters should be carried out to understand the true evolutionary variations in the Artemia resources.

\section{INTRODUCTION}

The Artemia species (The $2 \mathrm{n}$ ranged from 42 to 44) are widely distributed crustacean organisms that can inhabit hypersaline lakes and lagoons (Kong et al., 2019). Also, the osmotolerance of Artemia was extensively explained in many biological investigations (Gajardo and Beardmore, 2012; Kong et al., 2019).

The economic values of the Artemia in research laboratories and aquaculture as natural feeding stuff were extensively explained (Gajardo and Beardmore, 2012; Jamali et al., 2018).

Up to date, the true phylogenetic relationships among Artemia species especially the Egyptian Artemia resources are under debate (Saad et al., 2014; Eimanifar et al., 2015)

There are different laboratory techniques were applied for evaluating the biological differences among and within Artemia resources such as biometry, 
biochemical and molecular methods (Mahdhi et al., 2012; Saad et al., 2014; Saad and Elsebaie,2017).

Artemia is including a set morphologically similar species that mainly characterized by the criteria of reproductive isolation (Badaracco et al., 1995).

The body length character in Artemia (such as in A. sinica) was investigated and characterized by low heritability values (Kong et al., 2019). Therefore the morphological characterization might be not sufficient for inference the true evolutionary variations.

Regarding the molecular characterization, the accuracy in the Artemia species characterization was associated with the molecular identification system efficiency (Saad and Elsebaie 2017). Some molecular characterization systems such as $16 \mathrm{~S}$ ribosomal RNA, COI (Tizol-Correa et al., 2009; Maccari et al., 2013), AFLP (DeVos et al., 2013) and RAPD (Saad et al., 2014), were applied for estimating the genetic variability levels among and within many Artemia species and/or populations. Eimanifar et al. (2015) used COI barcoding system for exploring the population structures among some A. franciscana populations collected from different geographical localities of Great Salt Lake (the largest hypersaline lake in North USA).

Recently, Asem et al. (2019) used four molecular markers (Na+/K+ ATPase, COI, 16s rRNA and ITS1) to investigate the effects of environmental changes on Artemia genetic variability in the Urmia Lake.

Concerning the Artemia biological resources in Egypt, genetic diversity and speciation have not been evaluated in detail. In addition, no management strategies were applied for the utilization of Artemia genetic resources (Saad et al., 2014). The advantages of different molecular methods in detecting the molecular genetic variations among different animal taxa were discussed and confirmed (Tizol-Correa et al., 2009; Saad et al., 2013; Eimanifar et al., 2015; Saad et al., 2019).

The present study aimed to evaluate the evolutionary variations among some Egyptian brine shrimps comparatively with other Artemia species using some morphometric and molecular variations.

\section{MATERIALS AND METHODS}

\section{Artemia samples}

The Artemia samples were collected from three distantly Egyptian locations. These samples were A. parthenogenetica (MX from Alex. Salt marshes), A. salina (FA from El Fayoum Salt marshes) and A. salina (MN from Wadi El Natrun Valley). Also, two Artemia resources (A. franciscana from the Great Salt Lake, USA and A. parthenogenetica from China) were obtained from conservation of aquatic biological resources research group (DSR, King Abdulaziz University, KSA). The morphological characterization was carried out according to Clark and Bowen (1976).

\section{Morphometric analysis}

The morphometric parameters among the evaluated Artemia species were measured as described by Triantaphyllidis et al. (1997). A total of 12 morphometric parameters were scored for estimating the morphological variations among the evaluated Artemia species. The morphological parameters were the abdominal length (al), abdominal width (aw), distance between compound eyes (de), eye diameter (ed), length of furca (If), length of 1st antenna (la), total length (tl), head width (hw), ratio abdominal length/total length (B/A), ratio length of furca/total length (G/A), ratio 
head width/total length (I/A) and the length of 1st antenna/ total length (H/A). Also, the $(\lambda)$ Wilks' lambda is calculated. The $(\lambda)$ was given by the equation $\left(1-\eta^{2}\right)$, where $\eta^{2}$ is the ratio of the between-groups sum of squares to the total sum of squares. This parameter has represented the proportion of the total variance attributable to differentiate among the groups.

\section{Analysis of molecular variations among the evaluated Artemia species DNA extraction and PCR amplification}

The DNA samples were extracted from 15 Adults Artemia individuals from each evaluated Artemia species. DNA extraction and purification were carried out according to Badaracco et al. (1995) with some modifications (Saad et al., 2014).

\section{Analysis of ISSR markers}

Eleven ISSR primers were used to investigate the molecular variability among all the applied Artemia samples. The ISSR primer codes and sequences are presented in Table (1).

The PCR reactions were prepared as described by Saad et al. (2013). The PCR program consisted of one cycle for $3 \mathrm{~min}$. at $94^{\circ} \mathrm{C}, 35$ cycles for $\left(30 \mathrm{sec}\right.$. at $94^{\circ} \mathrm{C}, 45$ sec. at $44^{\circ} \mathrm{C}$ and $1 \mathrm{~min}$. at $72^{\circ} \mathrm{C}$ ) and one cycle for $15 \mathrm{~min}$. at $72^{\circ} \mathrm{C}$. The amplification products were separated on $1.5 \%$ agarose gels.

\section{Analysis of Artemia mitochondrial Cytochrome oxidase subunit I gene (COI) sequences}

A total of 30 Artemia COI gene sequence accessions were alignment and analyzed. Theses accessions were DQ119645, DQ119646 (Hou et al., 2006), DQ401269 (Tizol et al., 2009), DQ426856 (Munoz et al., 2008), DQ426832, DQ426834, DQ426841, DQ426846, DQ426848, DQ426849, DQ426854, DQ426855, DQ426856 \& DQ426858 (Munoz et al., 2008), from DQ426824 to DQ426826, from GU591380 to GU591384 (Munoz et al., 2010), from KC193638, KC193640 (Maccari et al., 2013), X69067.1 and NC_001620 (Perez et al., 1994), MK393285, MK393283, MK393289 and GU944723 (unpublished data obtained from NCBI).

Table 1: The ISSR primer codes, sequences, pattern evaluation, total number of generated bands and averages of band frequencies.

\begin{tabular}{lllll}
\hline Code & Sequence & pattern & TB & $\mathrm{BF} \pm \mathrm{SD}$ \\
\hline IT1 & CACACACACACACACAGT & good & 10 & $0.53 \pm 0.24$ \\
IT2 & CACACACACACACACACAAC & good & 7 & $0.88 \pm 0.31$ \\
IT3 & GAG GAG GAG GAG AG & good & 12 & $0.75 \pm 0.31$ \\
17898A & CAC ACA CAC ACA AC & good & 16 & $0.74 \pm 0.25$ \\
SAS1 & GTG GTG GTG GTGGC & good & 12 & $0.81 \pm 0.20$ \\
SAS3 & CAG GAG GAGGAGG & good & 8 & $0.75 \pm 0.28$ \\
HB12 & CACCACCACGC & good & 15 & $0.69 \pm 0.28$ \\
HB13 & GAG GAG GAG GC & good & 11 & $0.85 \pm 0.26$ \\
HB15 & GTG GTG GTG GC & good & 7 & $1 \pm 0$ \\
PT1 & GTGTGTGTGTGTGTGTC & $\underline{\mathcal{N C}}$ & - & - \\
HB8 & GAGAGAGAGAGAGG & $\mathrm{NC}$ & - & - \\
\hline
\end{tabular}

$\mathrm{TB}=$ Total bands, $\mathrm{BF}=$ average of band frequencies, $\mathrm{SD}=$ Standard deviation and $\mathrm{NC}=$ Unclear.

\section{Data analysis}

The morphometric variations and dendrogram construction were analyzed using the PAleontological Statistics, (PAST) Version 3.22 (Qyvind et al., 2001).

The ISSR profiles were scored and analyzed. The distance values were calculated using the Popgene. The results were introduced separately to two programs: Dendro UPGMA (a dendrogram construction utility) and PAST (Yeh and 
Boyle, 1997; Qyvind et al., 2001) for constructing the phylogenetic relationships among the evaluated Artemia species.

The COI sequences (617bp) analysis involved 30 nucleotide sequences. The COI sequences were aligned and the phylogenetic tree was re-constructed among the evaluated Artemia species using MEGA V6 (Tamura et al., 2013) based on the Maximum Likelihood method (MLM). Analysis of SNPs (single nucleotide polymorphisms) was carried out using DNAsp. (Ver.5.10.01).

\section{RESULTS AND DISCUSSION}

\section{Morphological variations}

The average values of the 12 morphometric characters (measured on the adult Artemia female samples) were calculated and analyzed. The interspecific variation values shared to the separation of the evaluated Artemia species were the total length (tl), abdominal length (al), the distance between compound eyes (de) and length of 1 st antenna (la). The lowest of these values were calculated for A. salina (FA).

The Ratio of the abdominal length/total length (B/A) values were succeeded in the discrimination among the evaluated Artemia samples comparatively with the other calculated Artemia body ratios (G/A, I/A and H/A) as presented in Table (2).

The Wilks' lambda $(\lambda)$ was calculated to explore the differences between group means for a particular combination of dependent variables (Figure1). The highest $(\lambda)$ value was calculated for (lf) character. On the other hand, the lowest $(\lambda)$ value (means more the variable) was calculated for (al).

Table 2: The ratio abdominal length/ total length, ratio length of furca / total length, ratio head width /total length and ratio length of 1st antenna/ total length within evaluated Artemia species.

\begin{tabular}{lllll}
\hline & B/A & G/A & I/A & H/A \\
\hline A. salina $(\mathrm{FA})$ & 0.3813 & 0.048 & 0.0938 & 0.0520 \\
A.parthenogenetica $(\mathrm{MX})$ & 0.5394 & 0.028 & 0.0552 & 0.0740 \\
A. salina $(\mathrm{MN})$ & 0.3374 & 0.036 & 0.0626 & 0.0495 \\
A.parthenogenetica $(\mathrm{SC})$ & 0.5375 & 0.028 & 0.0799 & 0.1066 \\
A. franciscana $(\mathrm{SA})$ & 0.45207 & 0.033 & 0.1066 & 0.1514 \\
\hline
\end{tabular}

$\mathrm{B} / \mathrm{A}=$ Ratio abdominal length/total length, G/A=Ratio Length of furca/total length, I/A=Ratio Head width / total length and $\mathrm{H} / \mathrm{A}=$ Length of 1 st antenna/ total length.

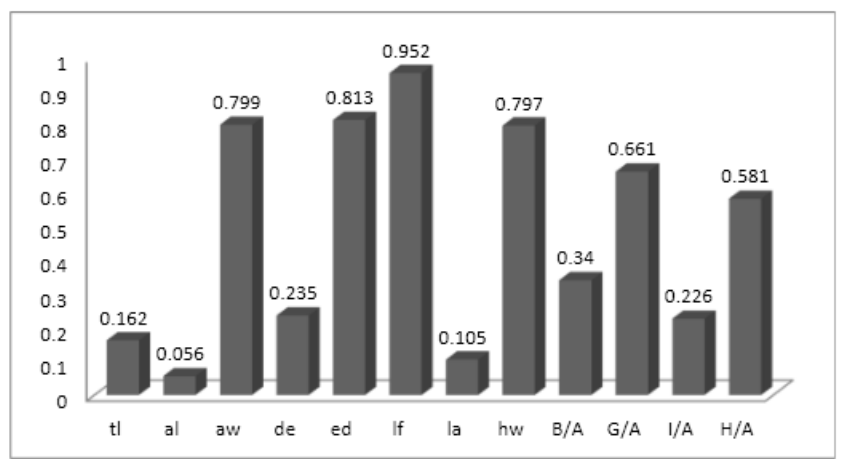

Fig.1: The Wilks' lambda $(\lambda=1-\eta 2)$ values of the selected morphometric female Artemia characters. $\mathrm{tl}=$ Total length, $\mathrm{al}=$ Abdominal length, $\mathrm{aw}=$ Abdominal width, de $=$ Distance between compound eyes, ed= Eye diameter, If= Length of furca, la= Length of 1 st antenna, hw=Head width, $\mathrm{B} / \mathrm{A}=$ Ratio abdominal length/ total length, $(\mathrm{G} / \mathrm{A})=$ Ratio Length of furca/total length, $(\mathrm{I} / \mathrm{A})=$ Ratio Head width/total length and H/A= Length of 1st antenna/ total length . 


\section{Analysis of ISSR markers}

Out of the 11 ISSR primers used, only two ISSR primers generated unclear banding patterns (PT1 and HB8). To evaluate the genetic variations and reconstructing the phylogenetic relations among the Artemia samples, the ISSR bands generated by the best ISSR primers (nine primers) were scored and analyzed. The total number of generated ISSR bands were detected and scored (98 bands). The number of generated ISSR bands were ranged from 7 (Primers IT2 and HB15) to 16 (primer 17898A). Also, the band frequency values were calculated for each selected ISSR primer in the evaluated Artemia species. The averages and standard deviations of these values were presented in Table (1). It was ranged from 0.53 (primer IT1) to 1 (primer HB15).

\section{The phylogenetic relationships among the evaluated Artemia species}

The phylogenetic relationships among the evaluated Artemia samples were constructed based on morphological (Figure 2a) and ISSR (Figure 2b) variations.

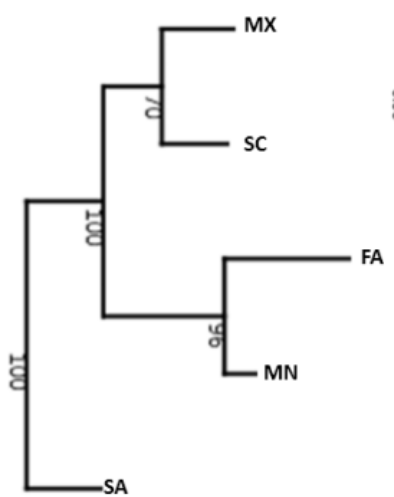

(a)

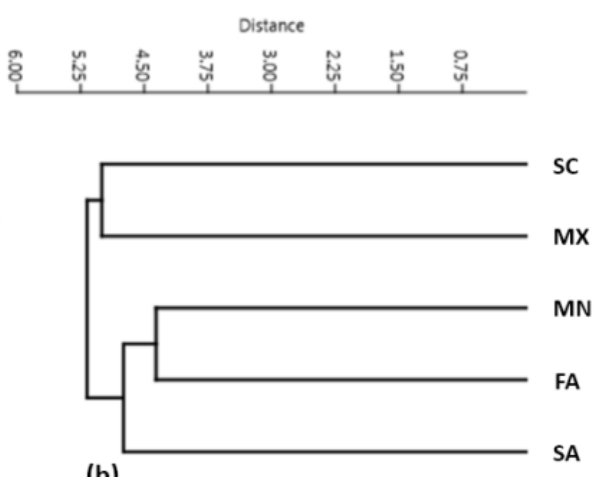

(b)

Fig. 2: The constructed dendrogram (UPGMA methods) among the evaluated Artemia species based on morphological (a) and ISSR (b) variations. $\mathrm{MX}=$ A.parthenogenetica, $\mathrm{MN}=$ A. salina, $\mathrm{FA}=$ A. salina, $\mathrm{SA}=$ A. franciscana, $\mathrm{SC}=$ A. parthenogenetica .

The relationships among evaluated Artemia species based on morphometric variations were similar that revealed from the molecular variations.

Concerning the molecular divergences, the lowest divergence value (distance $=$ 0.193) was observed between the two Egyptian A. salina biotypes (FA and MN). The genetic distance value between MX and SC Artemia species was lower than the distance between MX and all the other evaluated Artemia species. The similarity values among evaluated Artemia species were detected and presented in Table (3).

Table 3: The genetic identity (Above diagonal) and genetic distance (Below diagonal) among the evaluated Artemia species based on ISSR markers.

\begin{tabular}{llllll}
\hline & MN & FA & SA & SC & MX \\
\hline MN & & 0.824 & 0.749 & 0.785 & 0.770 \\
FA & 0.193 & & 0.797 & 0.770 & 0.774 \\
SA & 0.288 & 0.225 & & 0.788 & 0.775 \\
SC & 0.241 & 0.260 & 0.237 & & 0.801 \\
MX & 0.261 & 0.255 & 0.254 & 0.221 & \\
\hline
\end{tabular}

$\mathrm{MX}=$ A.parthenogenetica, $\mathrm{MN}=$ A. salina, $\mathrm{FA}=$ A. salina,

$\mathrm{SA}=A$. franciscana, $\mathrm{SC}=A$. parthenogenetica . 


\section{Inference of the evolutionary variations among the three Artemia species based on COI gene sequence variations}

A total of 30 COI DNA fragment sequences (617bp) in the three Artemia species (A. parthenogenetica, A. salina and A. franciscana) were analyzed.

The average values of $\mathrm{GC}(0.440), \mathrm{GC}_{2}(0.374)$ and $\mathrm{GC}_{3}(0.509)$ were calculated. The number of haplotypes $(\mathrm{h}=24)$, single nucleotide polymorphism $(\mathrm{SNPs}=186)$, estimates of haplotype diversity $(\mathrm{hd}=0.977)$, nucleotide diversity $(\mathrm{Pi}=0.1326)$, theta from polymorphic sites $(\Theta=0.0924)$, average number of nucleotide differences $(\mathrm{k}=81.814)$, conservation threshold $(\mathrm{CT}=0.8)$ and sequence conservation value $(\mathrm{Sc}=0.699)$ were calculated in all evaluated fragment sequences (Table 4).

The previous parameters were applied for estimating the genetic variations within each evaluated Artemia species. All DNA polymorphism were affected by the SNP value within each Artemia species. The highest single nucleotide polymorphism (SNPs=30) were calculated in A. salina.

These results were reflected also by the calculated genetic distance and haplotype diversity values (ranged between 0.8 to 1 ) within each Artemia species. The $\mathrm{GC}(0.415$ to 0.471$), \mathrm{GC}_{2}$ (0.314 to 0.370$)$ and $\mathrm{GC}_{3}(0.491$ to 0.540$)$ values were varied among the estimated $\mathrm{COI}$ sequences.

The estimated value of the shape parameter for the discrete Gamma Distribution is 0.3747. A discrete Gamma distribution was used to model evolutionary rate differences among sites. Mean evolutionary rates in these categories were $0.01,0.09,0.35,1.01,3.55$ substitutions per site. The nucleotide frequencies were $\mathrm{A}=22.05 \%, \mathrm{~T}=33.98 \%, \mathrm{C}=24.24 \%$, and $\mathrm{G}=19.72 \%$.

The phylogenetic relationships among the three Artemia species based on COI gene nucleotide variations were presented in Figure (3). The evolutionary history was inferred by using the Maximum Likelihood method.

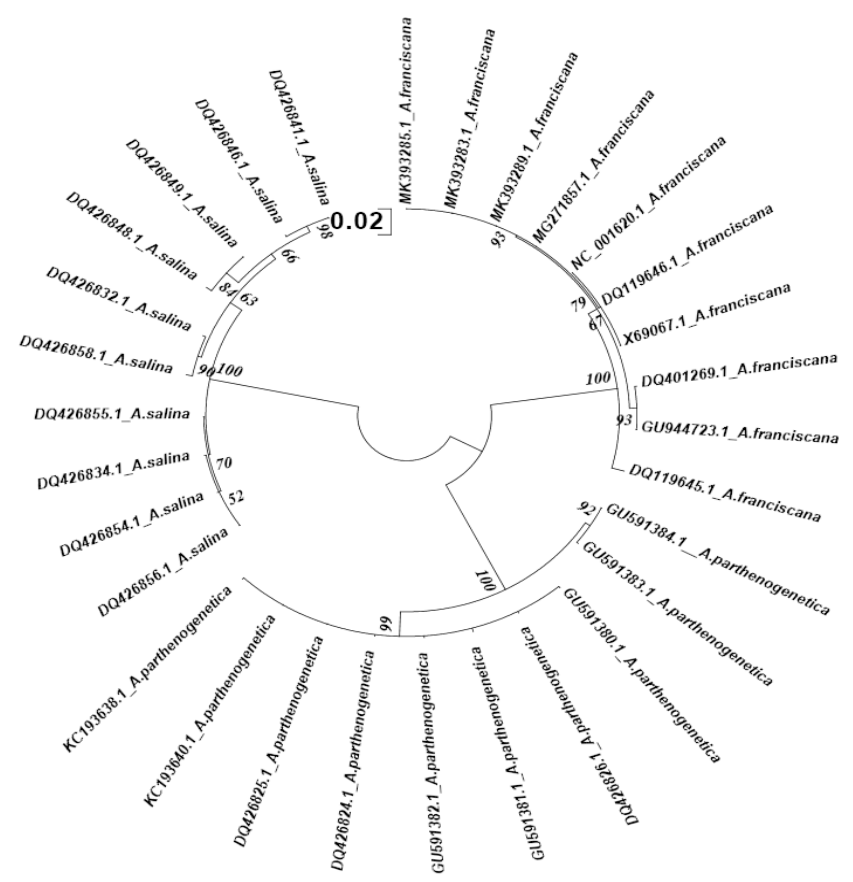

Fig. 3: Phylogenetic relationships among the Artemia species based on COI gene nucleotide variations (accessions were obtained from the NCBI). The evolutionary history was inferred by using the Maximum Likelihood method. 
Based on the consensus sequences, the distance value between A.salina and A.parthenogenetica is higher than the distance value between A.salina and A.franciscana. The evaluated COI sequence sites are beneficial in discriminating closely related Artemia species. The Variable COI consensus sequence sites among the three Artemia species are detected and presented in Figure (4).

The true evolutionary variations in the genus Artemia is still unclear and may be uncertain (Badaracco et al., 1995; Saad et al., 2014).

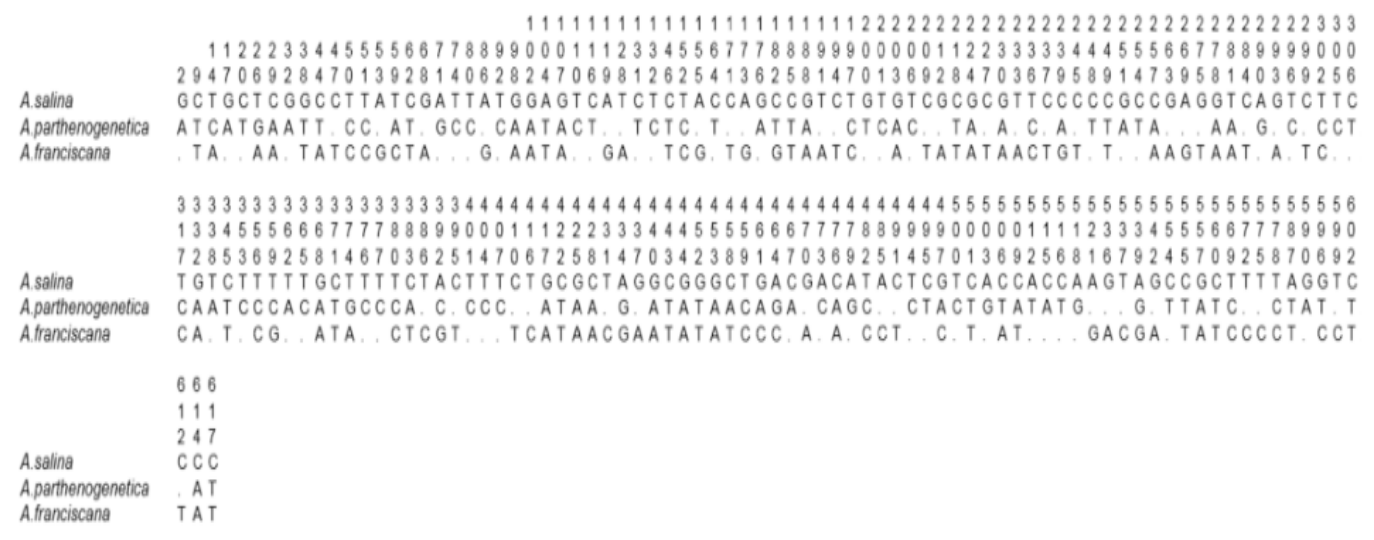

Fig. 4: The Variable COI consensus sequence sites among the three Artemia species.

Table 4: DNA polymorphism, sequence conservation and genetic distance values in eachevaluated Artemia species .

\begin{tabular}{lcccc}
\hline & A. salina & A. franciscana & A.parthenogenetica & Total \\
\hline SNP & 30 & 16 & 22 & 186 \\
GC & 0.471 & 0.415 & 0.433 & 0.440 \\
GC $_{2}$ & 0.437 & 0.314 & 0.370 & 0.374 \\
$\mathbf{G C}_{3}$ & 0.540 & 0.491 & 0.496 & 0.509 \\
Pi & 0.0182 & 0.0068 & 0.0106 & 0.1326 \\
$\mathbf{\theta}$ & 0.0177 & 0.0093 & 0.0129 & 0.0924 \\
K & 11.267 & 4.200 & 6.578 & 81.814 \\
H & 10 & 5 & 9 & 24 \\
Hd & 1 & 0.8 & 0.978 & 0.977 \\
SC & 0.951 & 0.974 & 0.964 & 0.699 \\
CT & 0.8 & 0.8 & 0.8 & 0.8 \\
Dist. & 0.019 & 0.007 & 0.011 & 0.16 \\
\hline
\end{tabular}

$\mathrm{SNP}=$ Single nucleotide polymorphism, $\mathrm{Pi}=$ nucleotide diversity, $\Theta=$ Theta from site, $\mathrm{K}=$ Average number of nucleotide differences, hd= Haplotype diversity= (hd), SC= Sequence conservation, CT= Conservation threshold and $\mathrm{D}=$ Distance value within each evaluated Artemia species

Some investigations recommended the analysis of different morphometric characters for exploring the evolutionary variations among different Artemia species such as in A. sinica and A. franciscana (Camargo et al., 2003; Kong et al., 2019). Other studies used some biochemical analysis for differentiating the Artemia resources (Ruiz et al., 2008).

In recent years attempts were made to correlate some Artemia species and/or populations living in different ecological locations around the world via molecular techniques (Eimanifar et al., 2015; Dunga et al., 2019). Generally, the evaluation of Artemia biodiversity through viable, simple and proper techniques such as ISSR is required as a basic step for good management through conservation of Artemia resources. 
In the present study, the combination between the molecular and the morphological characterization was applied to infer the evolutionary variations in the evaluated Artemia resources. The optimal discriminant variables for all the evaluated Artemia species were the total length, abdominal length, the distance between compound eyes and length of 1st antenna. However, only abdominal length and antenna length were recommended for discriminating among some Colombian $A$. franciscana populations. Measuring the left setae and antenna length were suitable for characterizing the Artemia males (Camargo et al., 2003).

The Wilks' lambda (as statistical parameters in multivariate analysis of variance) was applied in the present study for exploring the differences among the means of the estimated morphometric measurements. The highest $(\lambda)$ value was calculated for the (lf) character. On the other hand, the lowest $(\lambda)$ value (means more the variable) was calculated for (al). Application of these statistical parameters in similar data feature on some Artemia species (A. franciscana, A. tunisiana and A. urmiana) was recommended for Artemia species discrimination. These parameters could be informative in the classic method of Artemia morphological identification such as in Triantaphyllidis et al. (1997). Some morphological character variations might be affected by environmental conditions. Some investigations observed that the genetic variations among Artemia species might be correlated to geographical ranges and habitat heterogeneities of the investigated locations (Eimanifar et al., 2015).

The recommended Artemia morphometric characters provided suitable and informative classification methods when combined with the developed molecular markers. The divergence levels that detected in the present study between $A$. franciscana and the other evaluated Artemia species (A.salina and A.parthenogenetica) were confirmed also in some previous studies using other molecular techniques such as Random Amplified Polymorphic DNA (Badaracco et al., 1995).

Most of the selected ISSR primers succeeded in generating informative markers for discrimination among all investigated Artemia species. This finding was supported by the calculated ISSR band frequencies. All the selected primers were recommended for exploring the speciation in the Artemia biological resources accept three primers, HB15 (because of the mean of BF equal1), PT1 and HB8 (due to smearing in banding pattern).

The detected distance values between the two A.salina samples ( $\mathrm{MN}$ and F) supporting that ISSR technique was useful for evolutionary investigation of populations belonging to the same species. The same conclusion could be revealed from the detected molecular variations between the two A.parthenogenetica samples (MX and SC).

For estimating the efficiency of ISSR markers the results were compared with the COI gene sequence variations among the three Artemia species. Detection of mitochondrial gene sequences (such as 16s rRNA and COI as a DNA barcoding system) were recommended for its successful application in estimating the genetic variations among different marine organisms (Pondella et al., 2003, Ulises et al., 2018; Saad et al., 2019). The results showed similar evolutionary variations among Artemia species. Based on the consensus sequences, the distance value between $A$. salina and $A$. parthenogenetica was higher than the distance value between $A$. salina and $A$. franciscana.

The detected COI sequence polymorphism within and among the evaluated Artemia species were affected by the numbers of SNPs (Saad and Elsebaie, 2017). The analysis of SNPs revealed from the detected molecular markers would allow for 
accurate Artemia species identification and exploring evolutionary variations. This observation was confirmed in other biological investigations in different biological taxa including different aquatic animal species (Saad and Elsebaie, 2017; Wenne, 2018).

On the other hand, the low level of haplotype diversity (hd=0.8) in the $A$. franciscana is due to low detected SNPs in the analyzed gene region.

The efficiency of ISSR technique in evaluating the evolutionary variations among and within different aquatic and terrestrial animal taxa was extensively confirmed (Saad et al., 2013; Eimanifar et al., 2015).

\section{CONCLUSION}

The calculation of $(\lambda)$ as a statistical parameter was recommended for Artemia species discrimination based on morphological variations. The lowest $(\lambda)$ value (means more the variable) was calculated for the abdominal length character. The ISSR has proven its ability to detect speciation in the Artemia resources. The benefits of using the ISSR system for evaluating the Artemia evolution and speciation are that the markers were easier to amplify and detected. The results will be helpful for the conservation of the evaluated Artemia species. More molecular markers such as COI, 16s rRNA and simple sequence repeats should be developed and combined with the morphological variations to understand the true evolutionary variations in Artemia biological resources.

\section{ACKNOWLEDGEMENT}

The authors acknowledge with thanks the Conservation of biological Aquatic resources research group, King Abdulaziz University, KSA, for obtaining $A$. parthenogenetica and A. franciscana samples and technical support.

\section{REFERENCES}

Asem, A.; Eimanifar, A.; Van-Stappen, G.; Sun, S. (2019). The impact of one-decade ecological disturbance on genetic changes: a study on the brine shrimp Artemia urmiana from Urmia Lake, Iran. PeerJ, 7: e7190.

Badaracco, G.; Bellorini, M. and Landsberger, N. (1995). Phylogenetic Study of Bisexual Artemia Using Random Amplified Polymorphic DNA. Journal of Molecular Evolution, 41: 150-154.

Camargo, W.N.; Ely, J.S. and Sorgeloos, P. (2003). Morphometric characterization of thalassohaline Artemia franciscana populations from the Colombian Caribbean. Journal Biogeogrraphy, 30: 697-702.

Clark, L.S. and Bowen, S.T. (1976). The genetics of Artemia salina. VII. Reproductive isolation. Journal of Heredity, 67: 385-388.

De-Vos, S.; Bossier, P.; Van-Stappen, G.; Vercauteren, I.; Sorgeloos, P.; Stappen, G.; Vercauteren, I.; Sorgeloos, P. and Vuylsteke, M. (2013). A first AFLP-Based Genetic Linkage Map for Brine Shrimp Artemia franciscana and Its Application in Mapping the Sex Locus. PLOS ONE, 8(3): e57585.

Dunga, N.; Olivier, C.; Stephanie, D.; Thomas, H.; Guy, S. and Peter, B. (2019). Identification of RNAi-related genes and transgenerational efficiency of RNAi in Artemia franciscana. Aquaculture, 501: 285-292. 
Eimanifar, A.; Marden, B.; Braun, M. and Wink, M. (2015). Analysis of the genetic variability of Artemia franciscana Kellogg, 1906 from the Great Salt Lake (USA) based on mtDNA sequences, ISSR genomic fingerprinting and biometry. Marine Biodiversity, 45:311-319.

Gajardo, G. and Beardmore, J. (2012). The brine shrimp Artemia: adapted to critical life conditions. Frontiers Physiology, 3:1-8.

Hou, L.; Bi, X.; Zou, X.; He, C.; Yang, L.; Qu, R. and Liu, Z. (2006). Molecular systematics of bisexual Artemia populations Aquacult. Res., 37 (7): 671-680.

Jamali, H.; Ahmadifard, N.; Noori, F.; Agh, N. and Gisbert, E. (2018). Improving co-feeding strategies for Neotropical green terror cichlid (Aequidens rivulatus) larvae with lecithin-enriched Artemia franciscana nauplii: Effects on survival, growth performance and body composition. Aquacult. Res., 49:3909-3918.

Kong, Z.; Kong, J.; Luan, S.; Zhang, Z.; Yu, C. and Luo, K. (2019). Genetic Parameters of Body Length and Response to Selection for Growth Across Four Generations of Artemia sinica. J. Ocean University of China, 18: 203-209.

Maccari, M.; Gomez, A.; Hontoria, F. and Amat, F. (2013). Functional rare males in diploid parthenogenetic Artemia. J. Evolutionary Biology, 26 (9): 1934-1948.

Mahdhi, A.; Messina, C.; Kamoun, F.; Santulli, A. and Bakhrouf, A. (2012). Determination of biological characteristics of Tunisian Artemia salina populations. Biologia, 67:143-150.

Munoz, J.; Gomez, A.; Green, A.; Figuerola, J.; Amat, F. and Rico, C. (2008). Phylogeography and local endemism of the native Mediterranean brine shrimp Artemia salina (Branchiopoda: Anostraca). Molecular Ecology, 17 (13): 31603177.

Munoz, J.; Gomez, A.; Green, A.J.; Figuerola, J.; Amat, F. and Rico,C. (2010). Evolutionary origin and phylogeography of the diploid obligateparthenogen Artemia parthenogenetica (Branchiopoda: Anostraca). PLoS ONE, 5(8): e11932.

Palumbi, S. (1996). Nucleic acids II: The polymerase chain reaction. In: Hillis D.M.; Moritz, C. and Mable, K. (eds). Molecular Systematics. Sunderland, MA. Sinauer Associates, Inc. pp. 205-247.

Perez, M.; Valverde, J.; Batuecas, B.; Amat, F.; Marco, R. and Garesse, R. (1994).Speciation in the Artemia genus: mitochondrial DNA analysis of bisexual and parthenogenetic brine shrimps. Journal of Molecular Evolution, 38 (2): 156-168.

Pondella, D.; Craig, M. and Franckc, J. (2003). The phylogeny of Paralabrax (Perciformes: Serranidae) and allied taxa inferred from partial 16S and 12S mitochondrial ribosomal DNA sequences. Molecular Phylogenetics and Evolution, 29:176-184.

Qyvind, H.; David, H. and Ryan, P. (2001). Past: Paleontological Statistics Software Package for Education and Data Analysis. Palaeontologia Electronica, 4: 9pp.

Ruiz, O.; Amat, F.; Saavedra, C.; Papeschi, A.; Cohen, R.; Baxevanis, A.; Kappas, I.; Abatzopoulos, T. and Navarr, J. (2008). Genetic characterization of Argentinean Artemia species with different fatty acid profiles. Hydrobiologia, 610:223-234.

Saad, Y. M.; Abuzinadah, O.; El-Domyati, F.M. and Sabir, J. M. (2012). Analysis of Genetic signature for some Plectropomus species based on some dominant DNA markers. Life Science Journal, 9:2370-2375. 
Saad, Y. M.; Sabir, J. and Abuzinadah, O. (2013). Development of ISSR and multiplex ISSR markers for reconstructing phylogenetic relations among some shrimp species. Life Science Journal, 10(4): 1316-1322.

Saad, Y. M.; Heba, E.A.; Neveen, H.M. and Hanaa, M. (2014). Reconstruction of phylogenetic relations among some Artemia species. Life Science Journal, 11:822-826.

Saad, Y. M. and Elsebaie, H.A. (2017). The efficiency of Cytochrome oxidase subunit 1 gene (Cox1) in reconstruction of phylogenetic relations among some Crustacean species. The 19th International Conference on Animal Production, Mating and Breeding (ICAPMB), (27-28 July), Istanbul, Turkey.

Saad Y. M.; Sabir J. and Eldomyati F.M. (2019). Molecular phylogeny of mullet fishes using Sox14 and COI gene sequence variations. Research Journal of Biotechnology, 14 (4):130-146.

Tajima, F. and Nei, M. (1984). Estimation of evolutionary distance between nucleotide sequences. Molecular Biology and Evolution, 1:269-285.

Tamura, K.; Stecher, G.; Peterson, D.; Filipski, A. and Kumar, S. (2013). MEGA6: Molecular Evolutionary Genetics Analysis Version 6.0. Molecular Biology and Evolution, 30: 2725-2729.

Triantaphyllidis, G.; Criel, G.; Abatzopoulos ,T. and Sorgeloos, P. (1997). International study on Artemia. LIII. Morphological study of Artemia with emphasis to Old World strains. I. Bisexual populations. Hydrobiologia, 357: 139-153.

Tizol-Correa, R.; Maeda-Martinez, A.; Weekers, P.H.; Torrentera, L. and Murugan, G. (2009). Biodiversity of the brine shrimp Artemia from tropical salterns in Southern Mexico and Cuba. Current Science, 96 (1): 81-87.

Ulises, R.; Menendez, F.; Cornejo, R.; Canales, R.; Velez-Zuazo, X. (2018). Fish DNA barcoding around large marine infrastructure for improved biodiversity assessment and monitoring. Mitochondrial DNA Part A, 26:1-6.

Valsala, G.; Sugathan, S. and Bharathan, H.(2015). Loss of indigenous brine shrimp Artemia parthenogenetica due to the invasion by American species Artemia franciscana at Thamaraikulam salt pan. Indian Journal Geo-marine Sciences, 44 (11): 1712-1715.

Wenne, R. (2018). Single nucleotide polymorphism markers with applications in aquaculture and assessment of its impact on natural populations. Aquatic Living Resources, 31: 1-17.

Yeh, F. and Boyle, T. (1997). Population genetic analysis of co-dominant and dominant markers and quantitative traits. Belgian Journal of Botany, 129:157.A joint Project Development by Francis C. Yeh and Rongcai Yang, University of Alberta and Tim Boyle, Centre for International Forestry Research. 